\title{
ChemComm
}

Check for updates

Cite this: Chem. Commun., 2019, 55, 1726

Received 14th December 2018 Accepted 8th January 2019

DOI: $10.1039 / \mathrm{c} 8 \mathrm{cc} 09915 d$

rsc.li/chemcomm

\section{Butterfly proboscis-inspired tight rolling tapered soft actuators $\dagger$}

\author{
Jeroen A. H. P. Sol, (D) a Akhil R. Peeketi, (D) ${ }^{\mathrm{b}}$ Nihit Vyas, (D) ${ }^{\mathrm{b}}$ \\ Albertus P. H. J. Schenning, (D) ${ }^{\text {ac }}$ Ratna K. Annabattula (D) ${ }^{* b}$ and \\ Michael G. Debije (D)*a
}

Liquid crystalline networks have been fashioned into thin films with tapered thicknesses, revealing the possibility of rolling up extremely tightly when triggered thermally or with light. Compared to the often limited bending shown previously in liquid crystal network actuators, these tapered films curl up several hundreds of degrees. Finite element results of simulated functionally graded thin films with tapered thicknesses corroborate well with experimental work.

The world of robotics is at a point of revolution-soft robots are poised to challenge for supremacy. In particular, in the fields of sustainable energy harvesting, ${ }^{1}$ personal comfort ${ }^{2}$ and biomedical engineering, ${ }^{3}$ these remotely-addressable yet compliant materials are making great strides towards becoming the constituents of choice for many smart devices to come. For soft robotics researchers, the natural world is a great source of inspiration. ${ }^{4-8}$ Nature provides insights into many of the solutions to engineering problems facing soft robotics today: microrobots based on bacteria ${ }^{9}$ and electrospun water strider legs ${ }^{10}$ are two examples of using natural blueprints to generate novel man-made devices.

One of the prime candidate materials for bio-inspired soft robots is liquid crystal networks (LCNs), which are densely crosslinked polymers built up of reactive self-assembling monomers ("reactive mesogens"). ${ }^{11,12}$ The significance of LCNs in these developments is largely based on their anisotropic thermomechanical behaviour-upon disordering, LCNs shrink along the molecular director $\mathbf{n}$, while they expand perpendicular to $\mathbf{n} .^{13}$ LCNs have been used to create biomimetic soft actuators:

\footnotetext{
${ }^{a}$ Laboratory of Stimuli-Responsive Functional Materials and Devices (SFD), Department of Chemical Engineering and Chemistry, Eindhoven University of Technology (TU/e), 5600 MB Eindhoven, The Netherlands. E-mail: m.g.debije@tue.nl

${ }^{b}$ Stimuli-Responsive Systems Laboratory, Department of Mechanical Engineering, Indian Institute of Technology Madras (IITM), 600036 Chennai, India.

E-mail: ratna@iitm.ac.in

${ }^{c}$ Institute for Complex Molecular Systems (ICMS), Eindhoven University of Technology (TU/e), 5600 MB Eindhoven, The Netherlands

$\dagger$ Electronic supplementary information (ESI) available. See DOI: 10.1039/c8cc09915d
}

caterpillar-like inching robots, ${ }^{6}$ Venus flytrap-inspired grippers, ${ }^{5}$ high-power seedpod actuators, ${ }^{7}$ or, inspired by the locomotion of micro-organisms, "microrobots". 14,15

Remarkably, all LCN-based actuators reported to date feature a uniform film thickness. Recently, a tapered paper-polyester bilayer was reported in which the tapered structure showed neat rolling and could be used to grapple objects. ${ }^{16}$ However, in this case, the polyester acted as a shape memory material, meaning that autonomous actuation can only be performed once-in contrast to actuators based on LCNs, which are known to exhibit reversible motion. ${ }^{13}$

Previously shown splay-LCNs of constant film thickness typically bend up to a full rotation at elevated temperatures. ${ }^{13}$ In this work, LCNs are made with a thickness taper along the length of the film, similar to the proboscis of butterflies, their feeding organ (see Fig. 1a for a comparison). ${ }^{17,18}$ At rest, it is coiled up under their head, taking up minimal space. In action, it can extend to the body length of the butterfly and is used to retrieve nectar from plants or nutrients from rotting fruits, depending on the species of butterfly. ${ }^{19}$ Our proboscis-inspired tapered actuators show tight bending behaviour unique in splay-LCNs, with up to $3 \frac{1}{2}$ rotations upon heating or exposure to light. This rolling is fully reversible to reveal a nearly straight polymer strip at room temperature. Finite element modelling (FEM) results correspond well with experimental results. This allows for reversing the roles in the future, where the finite element model can be harnessed to anticipate the response of yet unexplored LC alignments and film geometries. Eventually, this could find use in designing LCN actuators that perform complex functions in larger soft robotic assemblies, or possibly as stand-alone devices in mixing systems for microfluidics.

Tapered-thickness, splay-aligned LCN thin films are obtained by filling alignment cells with a liquid crystal mixture consisting of equal weight fractions of monoacrylate 1 and diacrylate crosslinker 2 (see Fig. 1d for structures, more experimental details in the ESI $\dagger$ ). Photo-activated actuation is achieved through the incorporation of azobenzene dye 3 , which covalently bonds to the LCN during photo-polymerisation. Photo-initiation of the free 

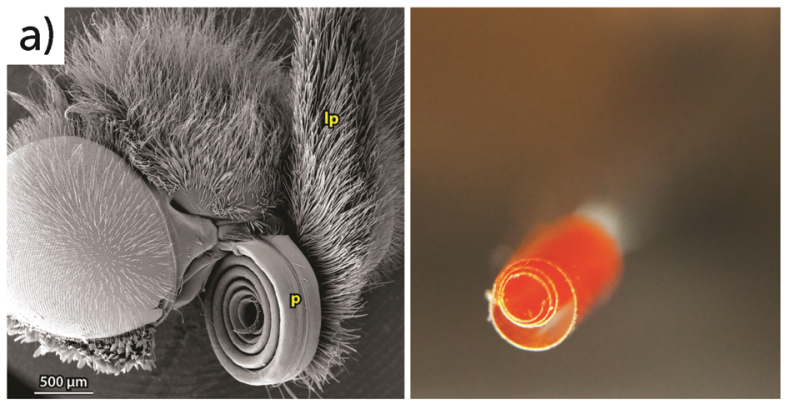

b)

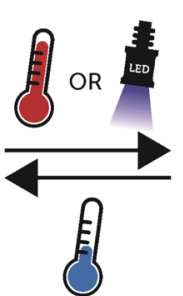

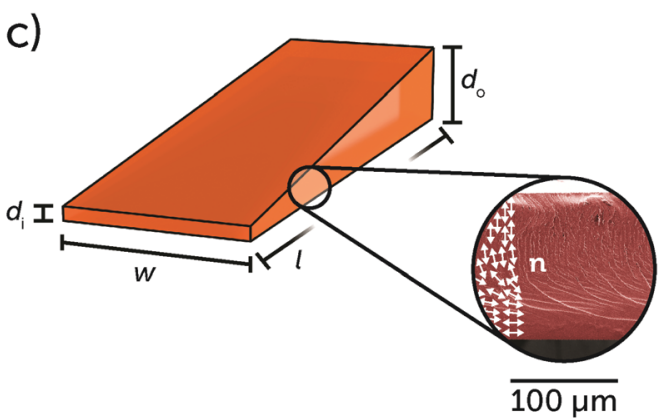

d)

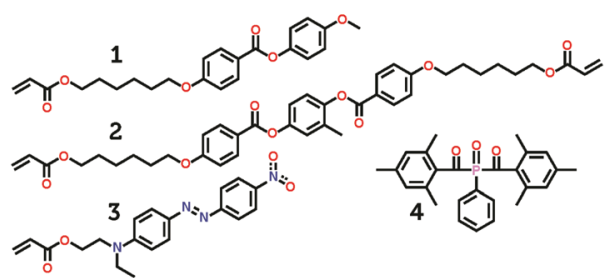

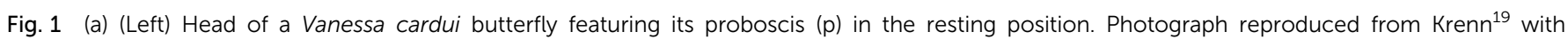

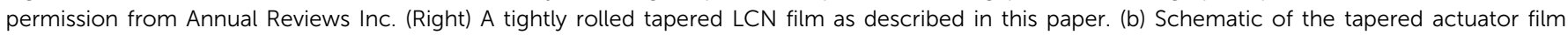

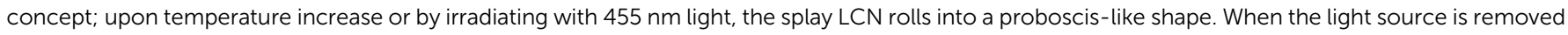

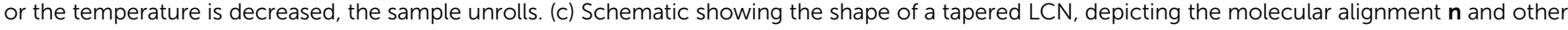

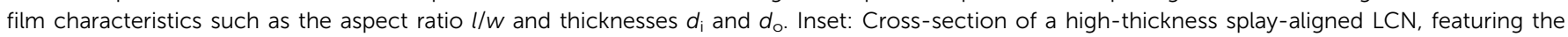

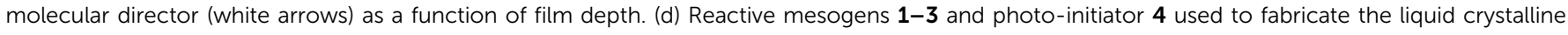
network actuator.

radical polymerisation is accomplished by 4 . The phase behaviour of the mixture was studied by differential scanning calorimetry (DSC) and polarised optical microscopy (POM). These results indicate an isotropic to nematic transition at $77{ }^{\circ} \mathrm{C}\left(T_{\mathrm{N}-\mathrm{I}}\right)$ when cooling, see Fig. S2 (ESI $\dagger$ ) for details.

Splay-aligned LC cells were made by sticking together glass with homeotropic and rubbed planar polyimide alignment layers facing each other. Spacer glue $(10 \mu \mathrm{m})$ and double-sided tape $(50 \mu \mathrm{m})$ were used to provide an LC alignment cell with a tapered cell gap. These cells were filled with the LC mixture at $90{ }^{\circ} \mathrm{C}$, above $T_{\mathrm{N}-\mathrm{I}}$. After filling, the temperature was lowered to $55{ }^{\circ} \mathrm{C}$, whereupon the LCs self-assembled into a nematic mesophase. After photo-initiated co-polymerisation to lock in the monomer's alignment, a thermal post-curing ensured maximum conversion of the acrylate groups (see Fig. S3, ESI, $\dagger$ for FT-IR data on $\mathrm{C}=\mathrm{C}$ conversion). DSC reveals that the phase transition peak at $T_{\mathrm{N}-\mathrm{I}}$ has disappeared after polymerisation, as expected (see Fig. S2, ESI $\dagger$ ). Cells were opened using a razor blade, revealing a thin film stuck to the glass coated with planar-aligned polyimide. Surface profilometry determined that film thicknesses were close to the spacer diameters chosen during cell manufacture. Furthermore, the thickness gradient between the thin and thick sides was linear. A polarised optical micrograph verified that the uniaxial nematic alignment was fixed during polymerisation, and dynamic mechanical analysis (DMA) revealed that the LCN has a broad glass transition range with the peak of $\tan (\delta)$ at around $65{ }^{\circ} \mathrm{C}\left(T_{\mathrm{g}}\right.$, see Fig. S4, ESI $\left.\dagger\right)$. Scanning electron micrographs confirm that even at film thicknesses exceeding $100 \mu \mathrm{m}$, splay alignment is retained (see Fig. 1c). The estimated ratios of penetration depth between homeotropic- and planar-aligned mesogens through the depth of the film depend on the film thickness (see Fig. S5, ESI $\dagger$ ).

Films were cut from the LCN as a strip with a $\Delta d=\left(d_{\mathrm{o}}-d_{\mathrm{i}}\right)=$ $20 \mu \mathrm{m}, d_{\mathrm{avg}}=25 \mu \mathrm{m}, l / w=4$ and the thickness taper $\Delta d$ in the same direction as the nematic director $\mathbf{n}$ (see Fig. 1c for a schematic image detailing these parameters). Upon removal of this film from the glass substrate, a free-standing film is obtained that shows no to little pre-bend, in which case the film slightly bends with the homeotropic side inward. This can be explained by the lower polymerisation temperature, which pre-empts the build-up of large thermal strains during polymerisation, and the thermal annealing, which also alleviates thermal stresses. ${ }^{20}$

The free-standing films were actuated in an oven with a window, through which photographs were recorded at set intervals. A digital temperature sensor recorded the temperature inside the oven at the same intervals. As the temperature was increased to $100{ }^{\circ} \mathrm{C}$, the film rolled up tightly, with a curvature of several hundreds of degrees. This is best visualised in a series of photographs, as in Fig. 2 (see the ESI, $\dagger$ Video S1, for a time-lapse video).

This behaviour can be explained with Timoshenko's model for bimetallic strips in mind, which correlates film thickness with the radius of curvature $\left(r_{\mathrm{C}}\right)$ for a given differential thermal strain. ${ }^{21}$ In splay-aligned films with planar anchoring on one face of the film and homeotropic on the other, curling is observed typically up to a full rotation at elevated temperatures. ${ }^{13}$ Later, the use of (reactive) azobenzene-containing dopants allowed for the LCN to be addressed and actuated with light. ${ }^{1,5,6,22-28}$ Compared to this earlier work the tapered films show an increasingly 


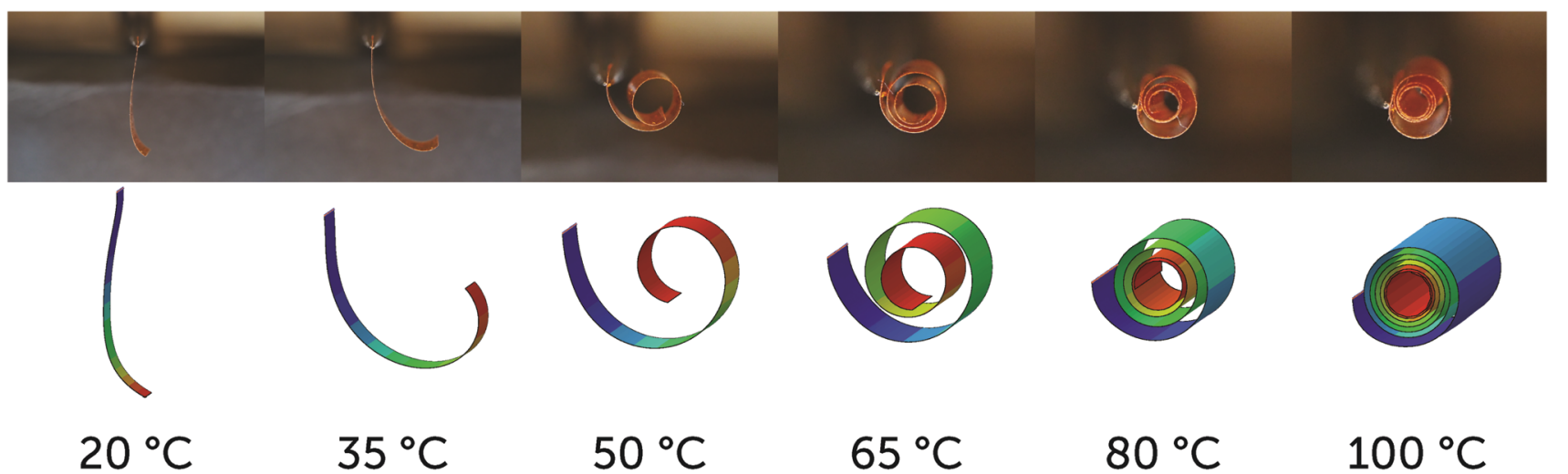

Fig. 2 Comparison of experimental thermomechanical bending results (top) with finite element method results (bottom) for a tapered splay-aligned LCN film at different temperatures. Coloured regions in the finite element results correspond to the increasing value of curvature $\left(1 / r_{\mathrm{C}}\right)$ from blue to red.

smaller $r_{\mathrm{C}}$ over their length, allowing them to effectively roll into themselves.

In order to better understand the bending behaviour of these tapered thickness LCNs, finite element simulations were performed. As described in more detail in the ESI, $\uparrow$ the tapered geometry along the length of the film is discretised using fournoded shell elements with composite sectional properties for the finite element model. The properties of the elastic moduli and the thermal expansion as a function of temperature were measured and used as input for the model (see Fig. S4 and S6, ESI $\dagger$ ). The finite element model incorporates the variation in the LC alignment direction through the depth of the film, thus making it a functionally graded system. The film is modelled as a three-layered system, in which each layer represents an area of the film with homeotropic, planar or intermediate $\left(45^{\circ}\right.$ tilt $)$ alignments. The relative thickness of the layers is programmed according to the depths found with scanning electron microscopy (SEM), as seen in Fig. S5 (ESI $\dagger$ ). A video of the simulated functionally graded layer (ESI, $\uparrow$ Video S2) shows that upon temperature increase tight rolling takes place.

There is a good correspondence between the predicted shapes and those achieved in practice (see Fig. 2), although the model somewhat overpredicts the bending behaviour of the film above $65^{\circ} \mathrm{C}$, the glass transition temperature. When plotting the inverse of the measured $r_{\mathrm{C}}$ (Fig. 3, extended data in Fig. S7, ESI $\dagger$ ), juxtaposed with the finite element result for $r_{\mathrm{C}}$, the same trend is seen even more clearly. We postulate that this is a result of a few factors. Firstly, the elastic moduli $\left(E^{\prime}\right.$ and $\left.E^{\prime \prime}\right)$ of the material diminish strongly above $T_{g}$, decreasing at least an order of magnitude. However, as predicted by the model, the main driver for rolling is the ratio between the elastic modulus parallel $\left(E_{11}\right)$ and perpendicular $\left(E_{22,33}\right)$ to n. From DMA results (Fig. S4, ESI $\dagger$ ) it is seen that the ratio between these moduli is not significantly influenced by the glass transition. In contrast, self-contact by the film might lead to friction that hampers tighter rolling. This is especially relevant as the material has its $T_{\mathrm{g}}$ around $65^{\circ} \mathrm{C}$, above which it is soft, which could promote surface adhesion. We postulate that above $T_{\mathrm{g}}$ a combination of this surface adhesion phenomenon with the lower absolute values of the elastic moduli is responsible for the overprediction by the model.

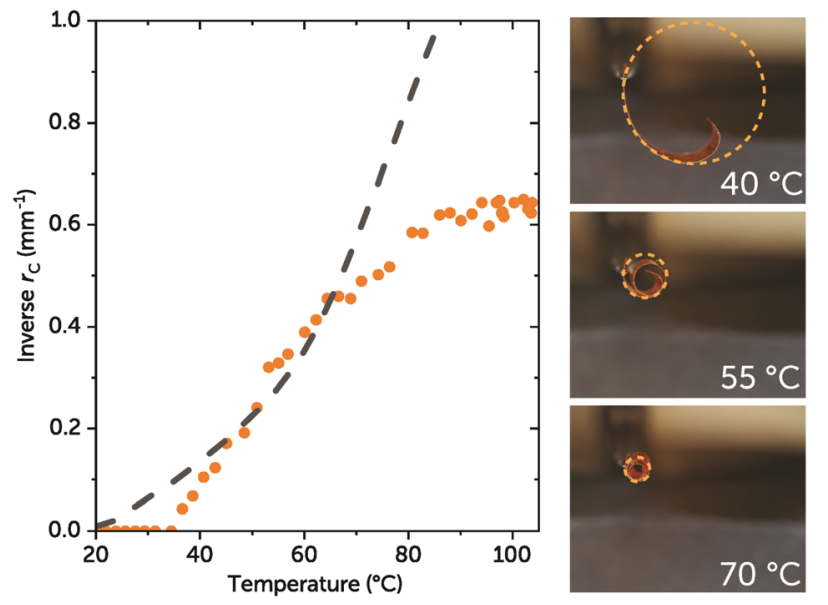

Fig. 3 Plot showing measured $r_{\mathrm{C}}$ for the LCN film from Fig. 2 at different temperatures (orange circles). The dashed grey line is composed of $r_{\mathrm{C}}$ values from finite element analysis. Photographs on the right side detail the measurement method for $r_{C}$.

So far, modelling results have pointed out that the number of rotations made during thermal actuation depends mainly on the difference between thermal expansion coefficients $(\Delta \alpha)$ parallel and perpendicular to $\mathbf{n}$ (see Fig. S6, ESI, $\uparrow$ for data on thermal strain).

To study the light response of the azobenzene-based LC material (compound 3, "disperse red 1 acrylate"), the film was irradiated with blue light $(\lambda=455 \mathrm{~nm})$, activating the trans-cis isomerisation. This photoactivated reaction releases heat, ${ }^{1}$ thereby inducing thermomechanical bending. As demonstrated in Fig. S8b and c (ESI $\dagger$ ), illumination from a single direction results in significant self-shading that prevents the film from bending fully into a rolled conformation. Turning on both light sources at once (Fig. 4) allows for otherwise shaded azobenzene molecules to be addressed, resulting in the formation of the anticipated tight curl. After turning off the light source, the film swiftly unbends, but a "post-bend" remains (ESI, $\dagger$ Video S3). We propose that this is due to vitrification during the backward motion, as actuation of the film occurs around the material's $T_{\mathrm{g}}$.

In summary, we have generated a series of LCN films from liquid crystalline networks that boast a tapered geometry, 

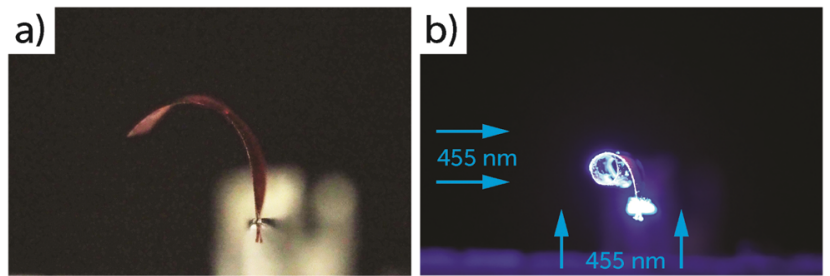

Fig. 4 Tapered splay-aligned film irradiated with $455 \mathrm{~nm}$ light: (a) no illumination and (b) illumination from left and underneath at a total intensity of about $620 \mathrm{~mW} \mathrm{~cm}^{-2}$.

with one side of the film thinner than the other. Actuation triggered by heat or light results in a smooth transition from fully extended to extremely tightly rolled morphologies, which are completely reversible and reproducible. Generation of actuators using tapered LCN films could allow more dramatic and consistent motions, allowing their application over a much broader range of devices to come. Our successful predictive model allows a wide exploration of potential actuation mechanisms to identify promising candidates to be produced experimentally.

The authors would like to express their gratitude towards Simon J. A. Houben (TU/e) for recording SEM pictures, and Marina Pilz da Cunha (TU/e) and Rob C. P. Verpaalen (TU/e) for results interpretation, discussion and DMA measurements. This research received funding from the Netherlands Organisation for Scientific Research (NWO) in the framework of the Innovation Fund Chemistry and from the Dutch Ministry of Economic Affairs in the framework of the PPP allowance.

\section{Conflicts of interest}

There are no conflicts to declare.

\section{Notes and references}

1 A. H. Gelebart, D. J. Mulder, M. Varga, A. Konya, G. Vantomme, E. W. Meijer, R. L. B. Selinger and D. J. Broer, Nature, 2017, 546, 632.

2 F. L. L. Visschers, M. Hendrikx, Y. Zhan and D. Liu, Soft Matter, 2018, 14, 4898.
3 M. Cianchetti, C. Laschi, A. Menciassi and P. Dario, Nat. Rev. Mater., 2018, 3, 143.

4 C. L. van Oosten, C. W. M. Bastiaansen and D. J. Broer, Nat. Mater., 2009, 8, 677.

5 O. M. Wani, H. Zeng and A. Priimagi, Nat. Commun., 2017, 8, 15546.

6 H. Zeng, O. M. Wani, P. Wasylczyk and A. Priimagi, Macromol. Rapid Commun., 2018, 39, 1700224.

7 S. J. Aßhoff, F. Lancia, S. Iamsaard, B. Matt, T. Kudernac, S. P. Fletcher and N. Katsonis, Angew. Chem., Int. Ed., 2017, 56, 3261 (Angew. Chem., 2017, 129, 3309).

8 T. J. Wallin, J. Pikul and R. F. Shepherd, Nat. Rev. Mater., 2018, 3, 84.

9 S. Palagi and P. Fischer, Nat. Rev. Mater., 2018, 3, 113.

10 F. Bai, J. Wu, G. Gong and L. Guo, ACS Appl. Mater. Interfaces, 2014, 6, 16237.

11 H. Zeng, P. Wasylczyk, D. S. Wiersma and A. Priimagi, Adv. Mater., 2018, 30, 1703554.

12 M. Lahikainen, H. Zeng and A. Priimagi, Nat. Commun., 2018, 9, 4148 .

13 G. N. Mol, K. D. Harris, C. W. M. Bastiaansen and D. J. Broer, Adv. Funct. Mater., 2005, 15, 1155.

14 H. Zeng, P. Wasylczyk, C. Parmeggiani, D. Martella, M. Burresi and D. S. Wiersma, Adv. Mater., 2015, 27, 3883.

15 D. S. Wiersma, S. Nocentini, C. Parmeggiani, D. Martella and D. Nuzhdin, in Optical Trapping and Optical Micromanipulation XIV, ed. K. Dholakia and G. C. Spalding, SPIE, 2017, p. 99.

16 W. Wang, C. Li, M. Cho and S.-H. Ahn, ACS Appl. Mater. Interfaces, 2018, 10, 10419.

17 H. W. Krenn and N. Mühlberger, Zool. Anz., 2002, 241, 369.

18 H. W. Krenn and N. P. Kristensen, Eur. J. Entomol., 2004, 101, 565.

19 H. W. Krenn, Annu. Rev. Entomol., 2010, 55, 307.

20 R. C. P. Verpaalen, M. G. Debije, C. W. M. Bastiaansen, H. Halilović, T. A. P. Engels and A. P. H. J. Schenning, J. Mater. Chem. A, 2018, 6, 17724 .

21 S. Timoshenko, J. Opt. Soc. Am., 1925, 11, 233.

22 T. J. White, J. Polym. Sci., Part B: Polym. Phys., 2018, 56, 695.

23 M. Yamada, M. Kondo, J. Mamiya, Y. Yu, M. Kinoshita, C. J. Barrett and T. Ikeda, Angew. Chem., Int. Ed., 2008, 47, 4986 (Angew. Chem., 2008, 120, 5064).

24 M. Hendrikx, B. Sirma, A. P. H. J. Schenning, D. Liu and D. J. Broer, Adv. Mater. Interfaces, 2018, 5, 1800810.

25 H. P. C. van Kuringen, Z. J. W. A. Leijten, A. H. Gelebart, D. J. Mulder, G. Portale, D. J. Broer and A. P. H. J. Schenning, Macromolecules, 2015, 48, 4073.

26 A. H. Gelebart, M. K. McBride, A. P. H. J. Schenning, C. N. Bowman and D. J. Broer, Adv. Funct. Mater., 2016, 26, 5322.

27 T. J. White, R. L. Bricker, L. V. Natarajan, N. V. Tabiryan, L. Green, Q. Li and T. J. Bunning, Adv. Funct. Mater., 2009, 19, 3484.

$28 \mathrm{~J}$. Lv, Y. Liu, J. Wei, E. Chen, L. Qin and Y. Yu, Nature, 2016, $537,179$. 